\section{Prompt diagnosis of ST-elevation myocardial infarction with papillary muscle rupture by point-of-care ultrasound in the emergency department}

\author{
Koon Ho Cheung ${ }^{1,2}$, Colin Graham Alexander ${ }^{1,2}$ \\ ${ }^{1}$ Accident and Emergency Department, Prince of Wales Hospital, Hong Kong \\ ${ }^{2}$ Accident and Emergency Medicine Academic Unit, The Chinese University of Hong Kong, Hong Kong
}

A previously healthy 61-year-old man presented to the emergency department with chest pain and dyspnoea for 6 hours. Examination revealed distress with an apical pansystolic murmur. Initial electrocardiogram showed sinus tachycardia and ST elevation in leads II, III, and aVF compatible with an inferior ST-elevation myocardial infarction. Point-of-care echocardiography in the emergency department showed a flail anterior mitral leaflet and severe mitral regurgitation, leading to a provisional diagnosis of papillary muscle rupture. Emergency cardiac catheterization showed 100\%, 80\%, and 70\% occlusion of the middle right coronary, left anterior descending, and left circumflex arteries, respectively. An emergency triple vessel coronary artery bypass grafting and mitral valve replacement was performed. Posteromedial papillary muscle rupture resulting in mitral regurgitation was confirmed intraoperatively. The patient recovered uneventfully. In the absence of primary percutaneous coronary intervention, thrombolysis decisions should be made with extreme caution if mechanical complications of ST-elevation myocardial infarction are suspected.

Keywords Myocardial infarction; Papillary muscle rupture; Point-of-care ultrasound; Echocardiography; Emergency service, hospital
elSSN: 2383-4625

Received: 13 August 2016

Revised: 6 September 2016

Accepted: 11 September 2016

Correspondence to: Koon Ho Cheung Accident and Emergency Department, Prince of Wales Hospital, 30-32 Ngan Shing Street, Shatin, New Territories, Hong Kong

E-mail: koonhocheung@yahoo.com.hk

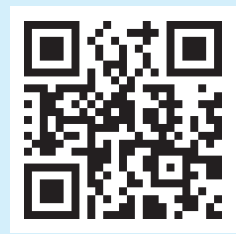

How to cite this article:

Cheung $\mathrm{KH}$, Alexander CG. Prompt diagnosis of ST-elevation myocardial infarction with papillary muscle rupture by point-of-care ultrasound in the emergency department. Clin Exp Emerg Med 2017;4(3):178-181

This is an Open Access article distributed under the terms of the Creative Commons Attribution Non-Commercial License (http:// creativecommons.org/licenses/by-nc/4.0/). 


\section{INTRODUCTION}

For patients with ST-elevation myocardial infarction (STEMI), the key management goals are early diagnosis and implementation of reperfusion therapy. A 12-lead electrocardiogram is critical for diagnosis of STEMI. For the treatment of STEMI with reperfusion, optimally performed primary percutaneous coronary intervention $(\mathrm{PCl})$ is preferable to fibrinolytic administration. ${ }^{1}$ However, fibrinolytic therapy remains the intended reperfusion strategy for patients who present to hospitals that cannot provide $\mathrm{PCl}$ or who require the procedure when the $\mathrm{PCl}$ cannot be performed. In considering whether fibrinolytic therapy will benefit a patient with myocardial infarction, the inclusion criteria and contraindications for fibrinolysis ${ }^{2}$ need to be evaluated and any mechanical complications must be identified. In patients presenting with STEMI, early detection of mechanical complications (e.g., papillary muscle rupture) is important because the established treatment for such conditions consists of $\mathrm{PCl}$ and surgery, and not a fibrinolytic agent. Point-of-care echocardiography allows prompt diagnosis of the mechanical complications of STEMI by the emergency department physician, as illustrated in the following case.

\section{CASE REPORT}

A previously healthy 61 -year-old man presented to the emergency department with chest pain and shortness of breath of six hours duration. He was distressed and sweated. His blood pressure was $116 / 74 \mathrm{mmHg}$, heart rate was 127 beats per minute, and oxygen saturation was $84 \%$ on room air. Examination revealed an apical pansystolic murmur and crepitations at both lung bases.

The initial electrocardiogram showed sinus tachycardia with ST elevation in leads II, III, and aVF, and reciprocal ST depression in leads V2-V5, I, and aVL, compatible with inferior STEMI (Fig. 1). In the emergency room, bedside transthoracic echocardiography showed a flail anterior mitral leaflet. The ejection fraction was moderately depressed, per visual inspection of the echocardiogram, and there was no pericardial effusion evident (Supplementary Video 1). The inferior wall was akinetic (Supplementary Video 2). Color flow showed a posteriorly directed jet of severe mitral regurgitation, and mild aortic regurgitation (Fig. 2 and Supplementary Videos 3 , 4). A provisional diagnosis of papillary muscle rupture was made.

The patient underwent emergency cardiac catheterization, which showed 100\% occlusion of the middle right coronary artery, $30 \%$ occlusion to the left main stem, $80 \%$ occlusion of the ostial left anterior descending artery, and 70\% diffuse occlusion of the left circumflex artery. He was transferred to the operating room for emergency surgery, which confirmed a posteromedial papillary muscle rupture resulting in mitral regurgitation. Coronary artery bypass grafting to left anterior descending artery, right coronary artery, and left circumflex artery, and mitral valve replacement were performed. The patient recovered uneventfully.

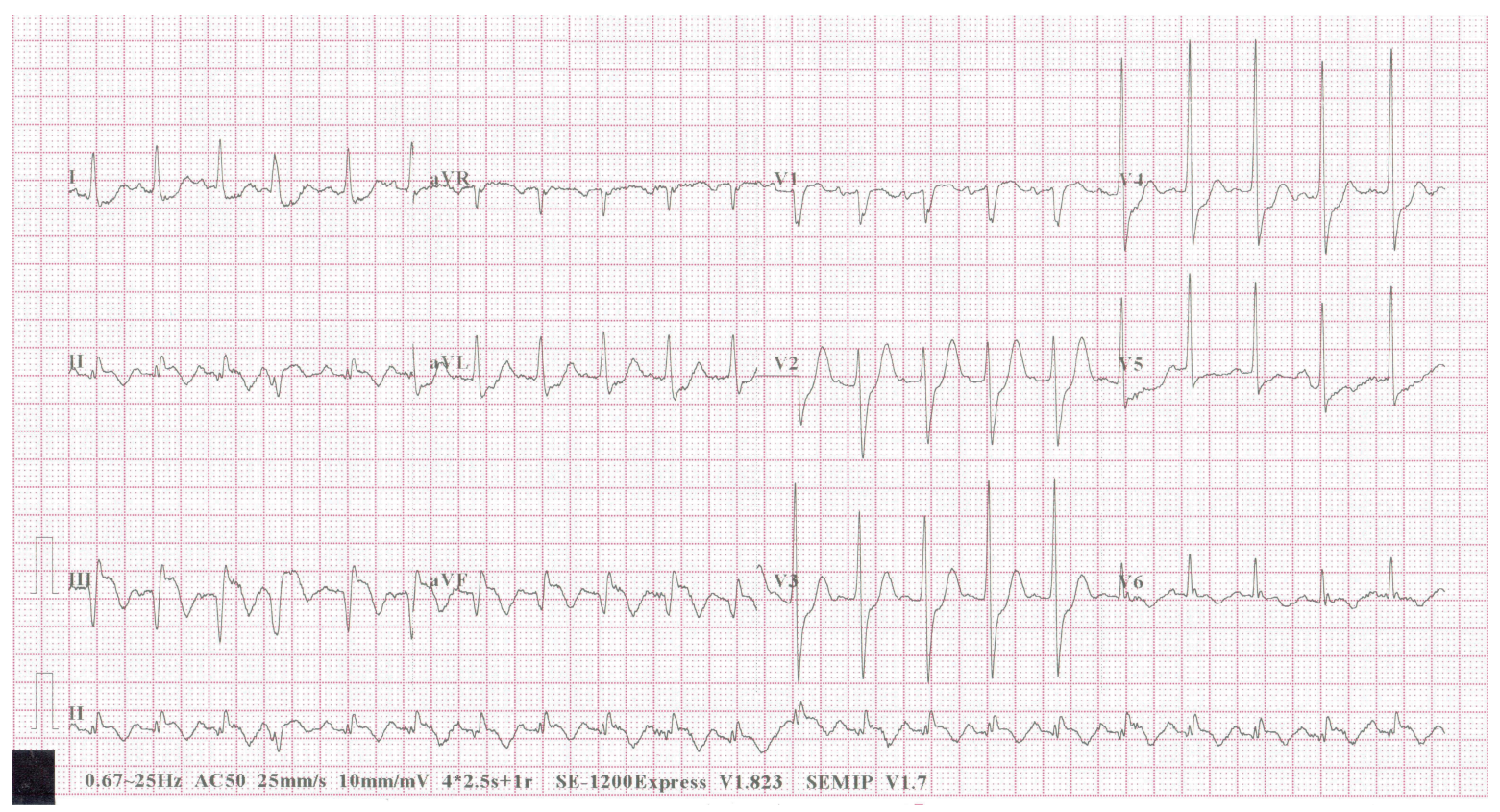

Fig. 1. Twelve-lead electrocardiogram shows ST elevations in leads II, III, and aVF, and ST depressions in leads V2-V5, I, and aVL, suggestive of inferior ST-elevation myocardial infarction. 

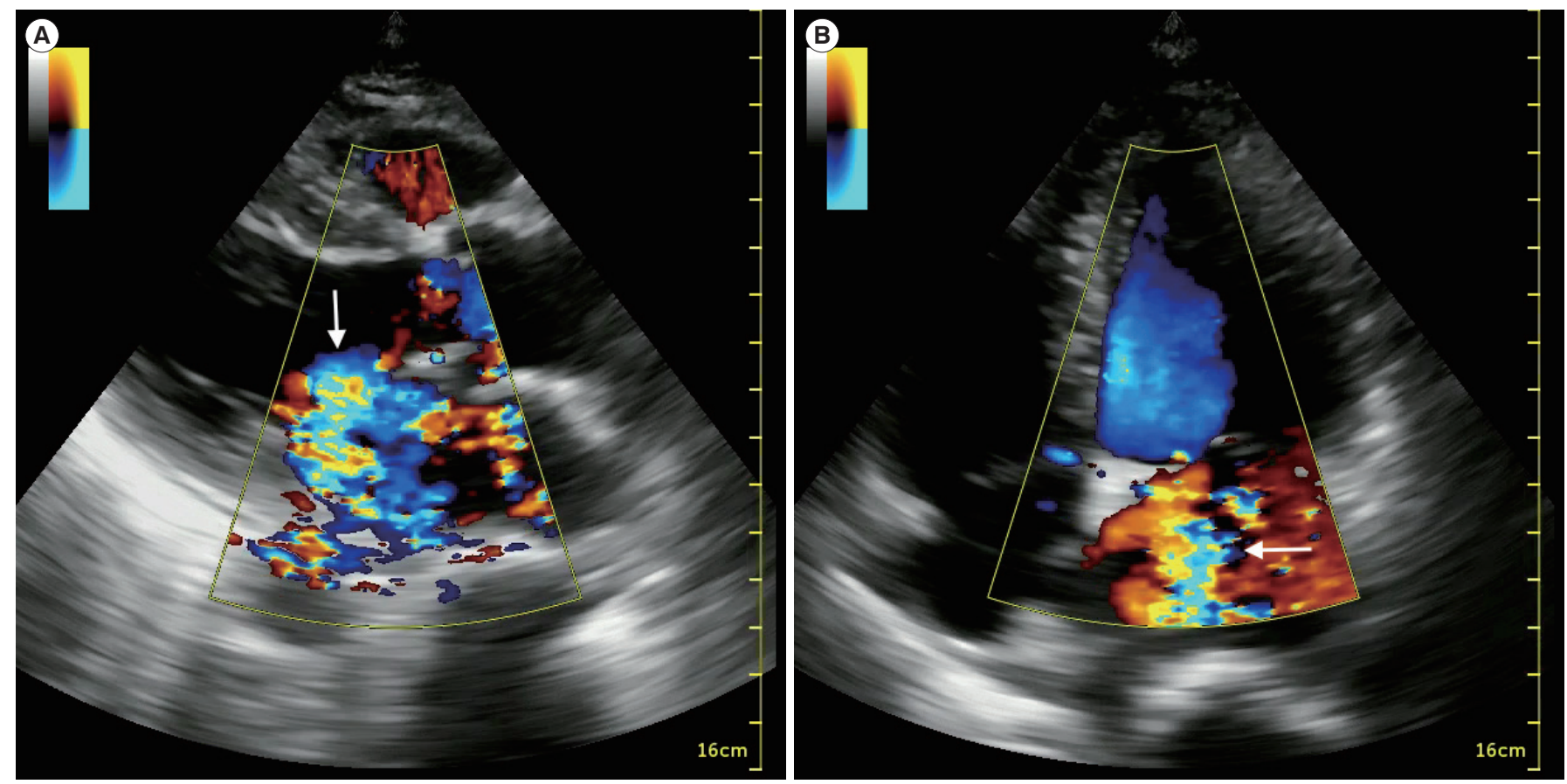

Fig. 2. (A) Transthoracic echocardiogram, parasternal long-axis view with color Doppler, demonstrating severe mitral regurgitation (arrow). (B) Transthoracic echocardiogram, apical-four-chamber view with color Doppler, demonstrating severe mitral regurgitation (arrow).

\section{DISCUSSION}

Papillary muscle ruptures with acute mitral regurgitation are uncommon $(<1 \%)$, but are often fatal complications secondary to myocardial infarction. The patient typically presents with features of myocardial infarction, a new apical systolic murmur, and commonly with cardiogenic shock. Differential diagnoses include ventricular septal defect and myocardial free wall rupture.

Transthoracic echocardiography makes it possible to visualize the regurgitant jet, the flail leaflet, and sometimes the ruptured papillary muscle, thus providing for an accurate diagnosis. Transesophageal echocardiogram can delineate the valves and papillary muscles better. The posteromedial papillary muscle, with its single blood supply, is more commonly involved in rupture than the anteromedial one, which has dual blood supplies. ${ }^{3}$ Both the anterior and posterior mitral leaflets are attached to both of the papillary muscles, so rupture of either papillary muscle can cause a flail of either leaflet. ${ }^{4}$ Treatment requires emergency surgery for mitral valve repair and revascularization. Medical treatment, without surgery, is associated with poor long-term survival. ${ }^{5}$

This case illustrates the importance of prompt bedside echocardiography in the emergency department for acute myocardial infarction. In many places in the world, thrombolytic therapy for STEMI is commonly administered when $\mathrm{PCl}$ is not available. Decisions regarding treatment with thrombolytics should be made with extreme caution if mechanical complications of STEMI are suspected. Point-of-care echocardiography by emergency physicians allows prompt diagnosis of mechanical complications in STEMI, which affects management strategy. This case also emphasizes the importance of access to cardiothoracic surgery in centers that can provide angiography.

\section{SUPPLEMENTARY MATERIALS}

Supplementary Videos are available from: https://doi.org/10.15441/ ceem.16.172.

Supplementary Video 1. Parasternal long axis view showing flail anterior mitral leaflet.

Supplementary Video 2. Parasternal short axis view showing akinetic inferior wall.

Supplementary Video 3. Parasternal long axis view with color Doppler showing severe mitral regurgitation.

Supplementary Video 4. Apical four-chamber view with color Doppler showing severe mitral regurgitation.

\section{CONFLICT OF INTEREST}

No potential conflict of interest relevant to this article was reported. 


\section{REFERENCES}

1. O'Connor RE, Al Ali AS, Brady WJ, et al. Part 9: acute coronary syndromes. 2015 American Heart Association guidelines update for cardiopulmonary resuscitation and emergency cardiovascular care. Circulation 2015;132(18 Suppl 2):S483-500.

2. CPR \& First Aid. Part 9: acute coronary syndromes. Web-based integrated 2010 \& 2015 American Heart Association guidelines for cardiopulmonary resuscitation and emergency cardiovascular care [Internet]. Dallas, TX: American Heart Association [cited 2016 Oct 14]. Available from: https://eccguide- lines.heart.org/index.php/circulation/cpr-ecc-guidelines-2/ part-9-acute-coronary-syndromes.

3. Fradley MG, Picard MH. Rupture of the posteromedial papillary muscle leading to partial flail of the anterior mitral leaflet. Circulation 2011;123:1044-5.

4. Czarnecki $A$, Thakrar $A$, Fang $T$, et al. Acute severe mitral regurgitation: consideration of papillary muscle architecture. Cardiovasc Ultrasound 2008;6:5.

5. Ling LH, Enriquez-Sarano M, Seward JB, et al. Clinical outcome of mitral regurgitation due to flail leaflet. $\mathrm{N}$ Engl J Med 1996;335:1417-23. 